\title{
Colistin resistance in KPC-producing Klebsiella pneumoniae strains from a high specialization rehabilitation facility
}

\author{
Roberta Migliavacca',Viola Repetto',Aurora Piazza', Elisabetta Nucleo', Elisabetta Abbaneo², \\ Silvana Telecco', Antonella Navarra', Laura Pagani' \\ I Sezione di Microbiologia, Dipartimento S. M. E. C.,Università di Pavia, Pavia \\ 2 Laboratorio di Microbiologia, I.R.C.C.S. Fondazione "Salvatore Maugeri", Pavia
}

Key words: Colistin resistance, Carbapenemases, Rehabilitation Facility

\begin{abstract}
Resistenza alla colistina in Klebsiella pneumoniae KPC-produttore in una struttura di riabilitazione di alta specializzazione
\end{abstract}

\section{SUMMARY}

The worldwide rapid spread of KPC carbapenemase-producing Klebsiella pneumoniae (KPC-Kp) represents an increasing problem in clinical settings. Reports on KPC-Kp epidemic spread in Italian hospitals began to appear since 2010; colistin (COL) represents one of the few remaining therapeutic options available for the treatment of such multi drug-resistant (MDR) pathogens.

Here we report the presence and diffusion of COL resistant KPC-Kp isolates from a High Specialization Rehabilitation Facility located in Northern Italy.

Species identification and antimicrobial susceptibilities were obtained by NBC46/NM40 Microscan panels (Siemens); imipenem, meropenem and ertapenem MICs were also evaluated by Etest and broth microdilution method; blaKPC-like genes PCR were performed.

PFGE (Xbal) was used to investigate clonal relatedness; epidemiological data were collected from the hospital database. Seventy-five carbapenem-resistant K. pneumoniae isolates were collected from the Fondazione S. Maugeri hospital during the period January-June 20II. Seven out of 75 MDR KPC-Kp isolates by Microscan System showed COL resistance (MIC >2 mg/L). Among them, 5/7 were collected from coma and 2/7 from cardiology and rehabilitation cardiology wards. Most of these strains were from urine (5/7); the remaining 2/7 were from blood and bronco-alveolar lavage. The $85.7 \%$ of the strains showed susceptibility to tigecycline and fosfomycin; 7 I.4\% only to gentamicina, $28.5 \%$ to trimethoprim/sulfamethoxazole and $14.2 \%$ to amikacin.

The PFGE profiles obtained analyzing 5/7 isolates from patients hospitalized from almost 10 days, showed clonal relatedness between $4 / 5$ isolates, thus confirming the high epidemic potential of almost one KPC-Kp clinical strain collected from 4 different wards. The emergence of COL resistance in KPC-Kp, dramatically reduces the available therapeutic options. These results underline the ability of a COL resistant KPC-producing clone to rapidly spread within this Rehabilitation Facility.

\section{INTRODUZIONE}

Klebsiella pneumoniae produttore di carbapenemasi di tipo KPC (KPC-Kp) è un patogeno in rapida diffusione a livello mondiale di grande impatto clinico-terapeutico.

Le strutture di riabilitazione e lungodegenza possono giocare un ruolo chiave nella diffusione di tali ceppi (6). I centri di alta specializzazione forniscono cure a pazienti affetti da malattie croniche che richiedono servizi medici ad hoc, prolungata ospedalizzazione, e/o utilizzo di devices invasivi (cateterizzazione, ventilazione forzata, ecc...). In tale tipologia di strutture, il rapido rilevamento su base fenotipica e la conferma molecolare di isolati multi-antibiotico-resistenti (MDR) carbapenemasi-produttori, sentinella di possibili evoluzioni epidemiche, possono risultare tuttavia particolarmente problematici da un punto di vista diagnostico-strumentale (5).

Stipiti KPC-Kp con fenotipo MDR mantengono sensibilità solo verso alcuni antibiotici, quali alcuni aminoglicosidi, colistina e tigeciclina (4); l'estrema limitazione delle opzioni terapeutiche determina elevati tassi di morbilità e mortalità infezione-associate.

Poichè la diffusione epidemica di KPC-Kp associata a multi-resistenza è stata descritta negli ospedali italiani a partire dal 2010, l'utilizzo di

\section{Corresponding author: Roberta Migliavacca}

Sezione di Microbiologia, Dipartimento S.M.E.C., Università degli Studi di Pavia

Via Brambilla, 74 - 27I00 Pavia - Tel.: 0382 984I43 - Fax: 03825484255

E-mail:r.miglia@unipv.it 
colistina (COL) riveste un ruolo terapeutico chiave. Rare risultano, attualmente, le segnalazioni in Italia di isolati carbapenemasi-produttori con resistenza estesa alla colistina (4).

Descriviamo la recente comparsa di tale resistenza in isolati KPC-Kp raccolti presso una struttura di riabilitazione di alta specializzazione del Nord Italia, che accoglie pazienti provenienti dall'intero territorio Nazionale.

\section{METODI}

Settantacinque isolati di $K$. pneumoniae carbapenemico- resistenti e KPC produttori (KPC-Kp) sono stati raccolti dalla Fondazione S. Maugeri nel periodo Gennaio-Giugno 2011. I test di sensibilità per i 75 ceppi sono stati effettuati utilizzando pannelli NBC46/NM40 Microscan System (Siemens); i risultati sono stati interpretati secondo le Linee Guida EUCAST 2011 (3).

La produzione di carbapenemasi è stata valutata fenotipicamente tramite test di Hodge Modificato. Tale test è stato effettuato seminando su $\mathrm{MH}$ agar il ceppo E. coli ATCC 25922 ( 0.5 McFarland), deponendo al centro della piastra un dischetto di ertapenem (ETP) e strisciando alcune colonie del ceppo in esame sull'inoculo di E. coli (a partire dal dischetto di ETP verso l'esterno). La distorsione dell'alone di inibizione della crescita di E. coli in corrispondenza del ceppo testato indica una produzione di carbapenemasi (2).

La presenza del gene blaKPC è stata confermata mediante amplificazione genica utilizzando i seguenti primer: Kpc1FW: ATG TCA CTG TAT CGC CGT CT; Kpc1RV: CAG GTT CCG GTT TTG TCT CC. Il protocollo di amplificazione (di un frammento genico di $710 \mathrm{bp}$ ) prevedeva una fase di denaturazione a $94^{\circ} \mathrm{C}(10 \mathrm{~min}) ; 30$ cicli di: $94^{\circ} \mathrm{C}(1 \mathrm{~min}), 50.5^{\circ} \mathrm{C}(1 \mathrm{~min}), 72^{\circ} \mathrm{C}(1 \mathrm{~min})$ ed infine un passaggio di estensione a $72^{\circ} \mathrm{C}(10 \mathrm{~min})$. La genotipizzazione è stata effettuata mediante PFGE con procedura Gene Path (Bio-Rad Laboratories): il DNA genomico è stato digerito con l'enzima XbaI ed i frammenti di restrizione separati in gel d'agarosio all' $1.0 \%$ in TBE $0.5 \mathrm{X}$ utilizzando l'apparecchio CHEF-DR II.

I concatenomeri del batteriofago $\lambda$ sono stati utilizzati come marker di peso molecolare.

I profili di restrizione sono stati analizzati con il software Fingerprinting II version 3.0 (Bio-Rad) utilizzando il metodo UPGMA. Per determinare il valore percentuale di similarità fra differenti profili è stato usato il coefficiente di correlazione Dice ed impostato un valore di tolleranza pari all' $1 \%$.

Per l'analisi di correlazione sono state considerate bande maggiori di $48 \mathrm{~Kb}$. Isolati che mostravano pattern di bande differenti fra loro per meno di sei frammenti e con similarità $\geq 75 \%$ sono stati considerati clonali.

I dati epidemiologici sono stati raccolti presso la banca dati ospedaliera.

\section{RISULTATI}

Durante il primo semestre 2011, dalla struttura di riabilitazione di alta specializzazione "Fondazione S. Maugeri" di Pavia, sono stati raccolti 75 isolati KPC-Kp.

Per 7/75 KPC-Kp, risultati MDR mediante il sistema Microscan, la resistenza interessava anche la colistina, con valori di MIC $>2 \mathrm{mg} / \mathrm{L}$.

Cinque di tali isolati (72\%) provenivano dall'unità risveglio, $1 / 7$ (14\%) dal reparto di cardiologia ed 1/7 (14\%) dal reparto di cardiologia riabilitativa. Nella maggior parte $(5 / 7 ; 72 \%)$ dei casi, i ceppi KPC-Kp COL-resistenti provenivano da campioni di urina, solo in $2 / 7(14 \%)$ dei casi erano stati isolati da emocoltura e bronco-aspirato (Grafici 1 A e B).

Cinque dei sette pazienti (71.7\%) erano stati sottoposti in precedenza a trattamento con colistina. Gli isolati sono risultati nell' $85.7 \%$ dei casi sensibili a fosfomicina e tigeciclina, nel $71.4 \%$ a gentamicina, nel $28.5 \%$ a trimetoprim/sulfametossazolo e nel $14.2 \%$ ad amikacina.

I valori di MIC per i carbapenemi sono risultati compresi tra $1 \mu \mathrm{g} / \mathrm{ml}$ e $8 \mu \mathrm{g} / \mathrm{ml}$ (Tabella 1). Dall'analisi PFGE eseguita su 5/7 isolati è emerso che $4 / 5$ di questi, provenienti da tre reparti differenti (unità risveglio, cardiologia, cardiologia riabilitativa) mostravano clonalità (Figura I).

\section{CONCLUSIONI}

La colistina rappresenta attualmente uno dei pochi antimicrobici attivi nei confronti di ceppi di $K$. pneumoniae MDR produttori di carbapenemasi. Tale antibiotico è pertanto frequentemente utilizzato, spesso in associazione con i carbapenemici, per il trattamento di infezioni gravi causate da ceppi MDR.

Un eccessivo o inadeguato uso di tale antibiotico può tuttavia determinare, in pazienti critici, l'insorgenza di ceppi resistenti alla colistina in corso di terapia (1).

L'emergere di una tale resistenza tra i ceppi KPCKp può annullare le opzioni terapeutiche.

I risultati sottolineano la diffusione di almeno due differenti cloni di K. pneumoniae KPC-produttori e COL resistenti in una struttura di riabilitazione del Nord Italia.

I risultati di tipizzazione molecolare hanno inoltre confermano l'elevato potenziale epidemico di un clone KPC-Kp COL resistente, diffusosi presso tre differenti reparti.

Il rafforzamento di misure igieniche mirate alla 
prevenzione/contenimento può contribuire efficacemente, soprattutto all'interno di alcune tipologie di strutture a rischio quali quelle di riabilita- zione/lungodegenza, a limitare la diffusione di ceppi problematici dal punto di vista clinico/epidemiologico qui segnalati.
$1 \mathrm{~A}$

Distribuzione dei 7 stipiti COL-resistenti per materiale

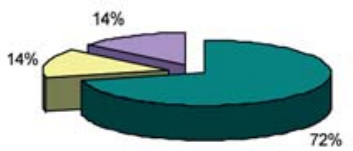

- Unita risveglio

$\square$ Cardiologia

$\square$ Cardiologia riabilitativa
1B

Distribuzione dei 7 stipiti COL-risistenti per campione biologico

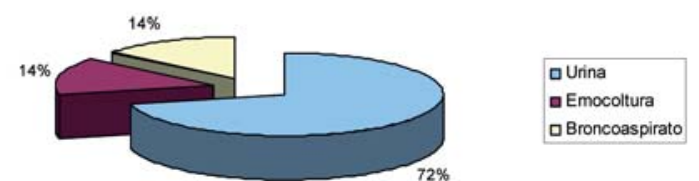

Grafico IA e IB. Distribuzione percentuale dei 7 stipiti COL-resistenti per materiale (IA) e campione biologico (IB).

Tabella I. Valori di MIC degli isolati K. pneumoniae in studio. L'interpretazione delle MIC (S, I, R) è in accordo ai breakpoint clinici EUCAST (Tabella V. I.3, 20 I I-0 I-05).

Antibiotici testati: imipenem (IMP), meropenem (MEM), ertapenem (ETP), amikacina (AK), gentamicina (CN), levofloxacina (LEV), ciprofloxacina (CIP), tigeciclina (TGC) and tobramicina (TO).

\begin{tabular}{|c|c|c|c|c|c|c|c|c|c|c|c|}
\hline \multirow[b]{2}{*}{ K. pneumoniae } & \multirow{2}{*}{$\begin{array}{c}\text { Data } \\
\text { isolamento }\end{array}$} & \multirow[b]{2}{*}{ AK } & \multirow[b]{2}{*}{ CIP } & \multirow[b]{2}{*}{ COL } & \multicolumn{3}{|c|}{ MIC $(\mu \mathrm{g} / \mathrm{ml}) /(\mathrm{S}, \mathrm{I}, \mathrm{R})$} & \multirow[b]{2}{*}{ FOS } & \multirow[b]{2}{*}{ GM } & \multirow[b]{2}{*}{ TIGE } & \multirow[b]{2}{*}{ TO } \\
\hline & & & & & ERT & IMP & MEM & & & & \\
\hline KI 40 MG 11 & $24 / 01 / 2001$ & $>16 \mathrm{R}$ & $>1 \mathrm{R}$ & $>2 \mathrm{R}$ & $>1 \mathrm{R}$ & $>8 \mathrm{R}$ & $>8 \mathrm{R}$ & $<=32 \mathrm{~S}$ & $>4 \mathrm{R}$ & $1 \mathrm{~S}$ & $>4 \mathrm{R}$ \\
\hline KI 51 MG 11 & 04/04/2011 & $>16 \mathrm{R}$ & $>1 \mathrm{R}$ & $>2 \mathrm{R}$ & $>1 \mathrm{R}$ & $8 \mathrm{I}$ & $8 I$ & $<=32 \mathrm{~S}$ & $<=2 \mathrm{~S}$ & $1 \mathrm{~S}$ & $>4 \mathrm{R}$ \\
\hline KI 52 MG 11 & 05/04/2011 & $>16 \mathrm{R}$ & $>1 \mathrm{R}$ & $>2 \mathrm{R}$ & $>1 \mathrm{R}$ & $>8 \mathrm{R}$ & $>8 \mathrm{R}$ & $<=32 \mathrm{~S}$ & $<=2 \mathrm{~S}$ & $1 \mathrm{~S}$ & $>4 \mathrm{R}$ \\
\hline KI 53 MG 11 & $09 / 05 / 2011$ & $<=8 \mathrm{~S}$ & $>1 \mathrm{R}$ & $>2 \mathrm{R}$ & $>1 \mathrm{R}$ & $>8 \mathrm{R}$ & $>8 \mathrm{R}$ & $>32 \mathrm{R}$ & $>4 R$ & $2 \mathrm{I}$ & $>4 \mathrm{R}$ \\
\hline KI 54 MG 11 & $27 / 06 / 2011$ & $16 I$ & $>1 \mathrm{R}$ & $>2 \mathrm{R}$ & $>1 \mathrm{R}$ & $>8 \mathrm{R}$ & $>8 \mathrm{R}$ & $<=32 \mathrm{~S}$ & $<=2 \mathrm{~S}$ & $0,5 \mathrm{~S}$ & $>4 \mathrm{R}$ \\
\hline KI 56 MG 11 & $30 / 06 / 2011$ & $>16 \mathrm{R}$ & $>1 \mathrm{R}$ & $>2 \mathrm{R}$ & $>1 \mathrm{R}$ & $>8 \mathrm{R}$ & $>8 \mathrm{R}$ & $<=32 \mathrm{~S}$ & $<=2 \mathrm{~S}$ & $1 \mathrm{~S}$ & $>4 R$ \\
\hline KL 58 MG 11 & $07 / 07 / 2011$ & $>16 \mathrm{R}$ & $>1 \mathrm{R}$ & $>2 \mathrm{R}$ & $>1 \mathrm{R}$ & $>8 \mathrm{R}$ & $>8 \mathrm{R}$ & $<=32 \mathrm{~S}$ & $<=2 \mathrm{~S}$ & $1 \mathrm{~S}$ & $>4 \mathrm{R}$ \\
\hline
\end{tabular}

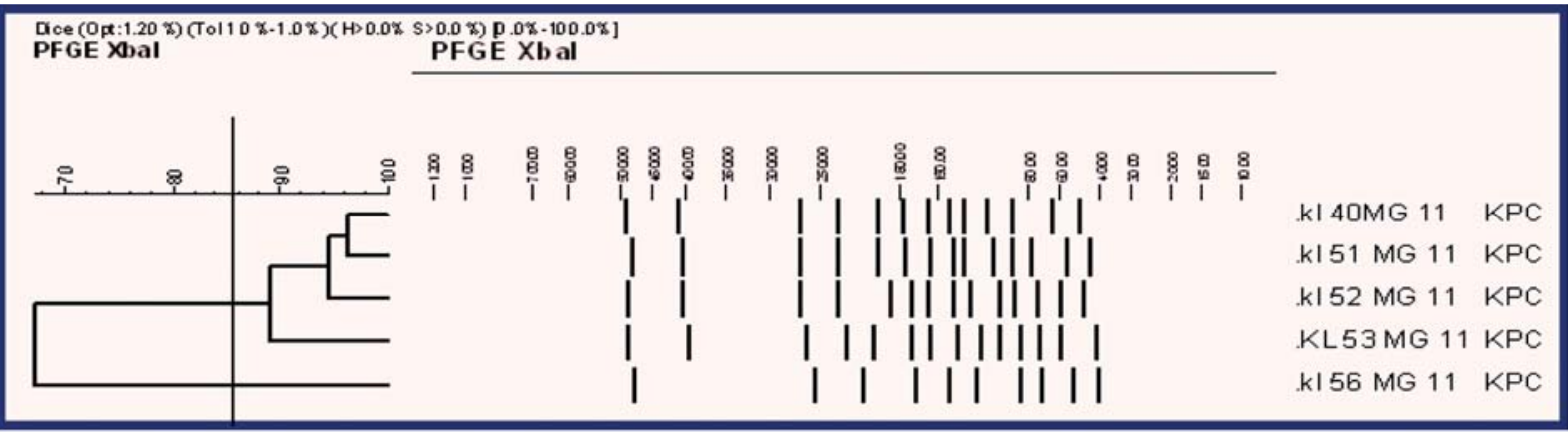

Figura I. Profili PFGE di cinque isolati di KPC-Kp colistina-resistenti dopo digestione con Xbal. La comparazione fra i profili è stata eseguita mediante software Fingerprinting II versione 3.0 (Bio-Rad).

\section{BIBLIOGRAFIA}

1. Antoniadou A, Kontopidou F, Poulakou G, et al. Colistin-resistant isolates of Klebsiella pneumoniae emerging in intensive care unit patients: first report of a multiclonal cluster. Journal of Antimicrobial Chemotherapy 2007; 59: 786-790

2. European Committee on Antimicrobial Susceptibility Testing (EUCAST). Version 1.3, 5 Jan 2011. 5Clinical and Laboratory Standard Institute, Wayne, PA

3. Grundmann H, Livermore DM, Giske CG et al. Carbapenem-non-susceptible Enterobacteriaceae in Europe: conclusions from a meeting of national experts. Euro Surveill 2010;15 (46): 19711.

4. Mezzatesta ML, Gona F, Caio C, et al. Outbreak of KPC-3-producing, and colistin-resistant, Klebsiella pneumoniae infections in two Sicilian hospitals. Clin Microbiol Infect 2011; 17 (9): 1444-7

5. Urban C., Bredford PA, Tukman $M$ et al. Carbapenem- resistant Escherichia coli harbouring Klebsiella pneumoniae carbapenemase beta-lactamases assiciated with long term care facilities. Clinical Infectiuos Diseases 2008; 46: 127-30

6. Won SY, Munoz-Price LS, Lolans K, et al. Emergence and rapid regional spread of Klebsiella pneumoniae carbapenemase-producing Enterobacteriaceae. Clinical Infectidious Diseases 2011. 53 (6): 532-540. 


\section{ERRATA CORRIGE}

Colistin resistance in KPC-producing Klebsiella pneumoniae strains from a high specialization rehabilitation facility

Roberta Migliavacca ${ }^{1}$, Viola Repetto ${ }^{1}$, Aurora Piazza ${ }^{1}$, Elisabetta Nucleo ${ }^{1}$, Elisabetta Abbaneo ${ }^{2}$, Silvana Telecco $^{2}$, Antonella $^{2}$ Navarra $^{2}$, Laura Pagani ${ }^{1}$

1 Sezione di Microbiologia, Dipartimento S. M. E. C., Università di Pavia, Pavia

2 Laboratorio di Microbiologia, I.R.C.C.S. Fondazione "Salvatore Maugeri", Pavia

MM 2012; 27 (1): 42

\section{SUMMARY}

Riga 16, "gentamincin" sostituisce "gentamicina"

Most of these strains were from urine (5/7); the remaining $2 / 7$ were from blood and bronco-alveolar lavage. The $85.7 \%$ of the strains showed susceptibility to tigecycline and fosfomycin; $71.4 \%$ only to gentamicin, $28.5 \%$ to trimethoprim/sulfamethoxazole and $14.2 \%$ to amikacin.

MM 2012; 27 (1): 44

BIBLIOGRAFIA

nel riferimento nr. 2 eliminare Clinical and Laboratory Standard Institute, Wayne, PA

2. European Committee on Antimicrobial Susceptibility Testing (EUCAST). Version I.3, 5 Jan 20I I. 5 